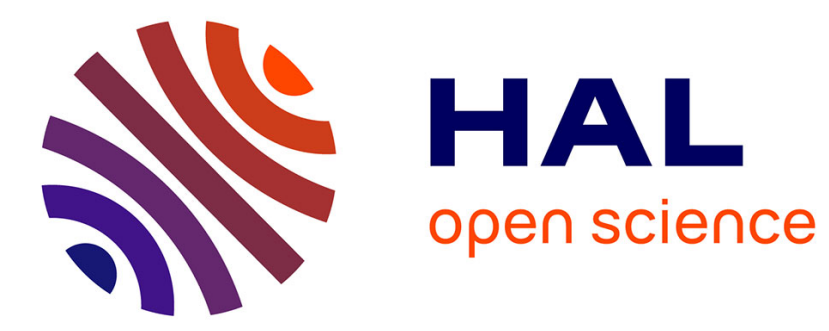

\title{
La pratique des activités physiques et sportives : les inégalités entre les filles et les garçons sont-elles plus réduites dans le cadre scolaire ?
}

Gilles Combaz, Olivier Hoibian

\section{- To cite this version:}

Gilles Combaz, Olivier Hoibian. La pratique des activités physiques et sportives : les inégalités entre les filles et les garçons sont-elles plus réduites dans le cadre scolaire ?. Carrefours de l'éducation, 2011, 32 (2), pp.167-185. 10.3917/cdle.032.0167 . hal-03354771

HAL Id: hal-03354771

https://hal.univ-lyon2.fr/hal-03354771

Submitted on 27 Sep 2021

HAL is a multi-disciplinary open access archive for the deposit and dissemination of scientific research documents, whether they are published or not. The documents may come from teaching and research institutions in France or abroad, or from public or private research centers.
L'archive ouverte pluridisciplinaire HAL, est destinée au dépôt et à la diffusion de documents scientifiques de niveau recherche, publiés ou non, émanant des établissements d'enseignement et de recherche français ou étrangers, des laboratoires publics ou privés. 


\title{
LA PRATIQUE DES ACTIVITÉS PHYSIQUES ET SPORTIVES : LES INÉGALITÉS ENTRE LES FILLES ET LES GARÇONS SONT-ELLES PLUS RÉDUITES DANS LE CADRE SCOLAIRE?
}

\author{
Gilles Combaz et Olivier Hoibian
}

Armand Colin | Carrefours de l'éducation

$2011 / 2-n^{\circ} 32$

pages 167 à 185

ISSN 1262-3490

Article disponible en ligne à l'adresse:

http://www.cairn.info/revue-carrefours-de-l-education-2011-2-page-167.htm

Pour citer cet article :

Combaz Gilles et Hoibian Olivier, « La pratique des activités physiques et sportives : les inégalités entre les filles et les garçons sont-elles plus réduites dans le cadre scolaire ? »,

Carrefours de l'éducation, 2011/2 n³2, p. 167-185. DOI : 10.3917/cdle.032.0167

Distribution électronique Cairn.info pour Armand Colin.

(c) Armand Colin. Tous droits réservés pour tous pays.

La reproduction ou représentation de cet article, notamment par photocopie, n'est autorisée que dans les limites des conditions générales d'utilisation du site ou, le cas échéant, des conditions générales de la licence souscrite par votre établissement. Toute autre reproduction ou représentation, en tout ou partie, sous quelque forme et de quelque manière que ce soit, est interdite sauf accord préalable et écrit de l'éditeur, en dehors des cas prévus par la législation en vigueur en France. II est précisé que son stockage dans une base de données est également interdit. 


\title{
La pratique des activités physiques et sportives: les inégalités entre les filles et les garçons sont-elles plus réduites dans le cadre scolaire?
}

\author{
- Gilles Combaz, Olivier Hoibian \\ gilles.combaz@univ-lyonz.fr \\ olivier.hoibian@wanadoo.fr
}

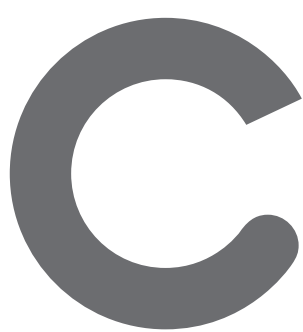
et article aborde la question des variations de la pratique des activités physiques selon le sexe en les resituant de manière plus large dans la perspective des inégalités entre filles et garçons à l'école. Ces dernières ont fait l'objet, en France, depuis le début des années 1980, de plusieurs dispositifs institutionnels visant une meilleure égalité. Le dernier en date est la convention interministérielle signée pour la période 2006-2011 ${ }^{(1)}$. Le site Internet du ministère de l'Éducation nationale comprend une rubrique consacrée spécifiquement à la question de l'égalité entre les sexes. On peut y lire que: «l'égalité des filles et des garçons constitue pour l'Éducation nationale une obligation légale et une mission fondamentale» (Ministère de l'Éducation nationale, 2010). Incontestablement, il s'agit d'une question importante mais la succession des nombreux dispositifs institutionnels proposés dans un laps de temps assez court ne laisse-t-elle pas supposer que les principaux objectifs n'ont pas été atteints? Existe-t-il des résistances qui freinent la mise en œuvre effective des mesures concernées?

Si les textes officiels garantissent une égalité de droits entre les sexes, ils n'impliquent pas automatiquement une égalité dans les faits. Et de ce point de vue, il convient de distinguer égalité de droits, égalité de traitement et égalité de résultats (Duru-Bellat, 2002). En ce qui concerne l'égalité de résultats, les données disponibles montrent clairement qu'à tous les niveaux du système éducatif, les filles, en règle générale, réussissent mieux que les garçons (Rosenwald, 2006 ; ministère de l'Éducation nationale, 2010). Une

(1). Convention pour l'égalité entre les filles et les garçons, les femmes et les hommes, dans le système éducatif, Bulletin officiel de l'Éducation nationale, n 5 du $1^{\text {er }}$ février 2007, p. 254258. 
partie de ces derniers serait, selon certains auteurs, dans une situation scolaire préoccupante. Ils proposent des pistes de réflexion pour que l'on « sauve les garçons » (Auduc, 2009). Si les filles dominent en termes de réussite, elles ne sont pas pour autant traitées à égalité avec les garçons au sein de l'école. C'est ce que révèlent de nombreux travaux centrés sur la question des inégalités scolaires sexuées. Plusieurs revues de la littérature montrent que des inégalités sont repérables dans trois domaines: les interactions entre le maître et les élèves en classe; les contenus d'enseignement et les manuels scolaires; l'orientation scolaire et professionnelle (Duru-Bellat, 1995; Petrovic, 2004). Même si quelques évolutions se dessinent, des travaux récents révèlent que les progrès sont assez lents. En ce qui concerne les interactions en classe, on observe encore un déséquilibre en faveur des garçons (Mosconi, 2001; Trottin, Cogérino, 2009). Le contenu de certains manuels scolaires à l'école primaire reste relativement sexiste (Brugeilles et al., 2009). Les contenus d'enseignement en éducation physique et sportive au lycée ont tendance à avantager les garçons (Combaz, Hoibian, 2008). Enfin, les procédures d'orientation scolaire contribuent encore à une répartition assez sexuée des filles et des garçons au sein des différentes filières scolaires (Vouillot, 2007). Les jeunes filles qui s'engagent dans des filières dites « masculines» éprouvent des difficultés bien plus importantes que les garçons insérés dans des filières « féminines » (Lemarchant, 2007).

Dans cet article, nous proposons d'aborder la question des inégalités entre les sexes à l'école sous un angle très peu souvent traité: la pratique des activités physiques et sportives. En France, au sein du système éducatif, dans le second degré, les élèves ont l'occasion de pratiquer des activités physiques selon deux principales modalités: dans le cadre des enseignements obligatoires d'éducation physique et sportive (EPS) et, de manière facultative, dans le cadre du sport scolaire.

Si l'EPS a fait l'objet de nombreux travaux consacrés aux inégalités entre filles et garçons ${ }^{(2)}$, ce n'est pas le cas du sport scolaire. Les recherches réalisées dans ce domaine-là sont relativement rares.

\section{Points d'appui pour la recherche}

\section{L'éclairage conjoint de la sociologie de l'éducation et de la sociologie du sport}

De par ses spécificités (que nous développerons plus loin), le sport scolaire se situe à l'interface entre l'école et la sphère des pratiques physiques extrascolaires. L'implication des filles et des garçons en son sein et les inégalités qui peuvent y

(2). Cf. entre autres: Louveau, Davisse (1998), Cogérino (2005), Vigneron (2006), Trottin, Cogérino, (2009). 
être associées seront étudiées en prenant appui sur des travaux issus de la sociologie de l'éducation et de la sociologie du sport.

Postuler, comme le laisse entendre le titre de cet article, que les inégalités de pratiques physiques selon le sexe pourraient être plus réduites au sein de l'école suppose que cette dernière serait capable d'infléchir des tendances qui lui sont externes. Autrement dit, la question est de savoir si l'école serait véritablement en mesure de neutraliser - voire de contrer - les effets puissants de la socialisation primaire qui prédispose très inégalement les filles et les garçons à la pratique des activités physiques. Cette première interrogation renvoie plus largement au rôle que l'école joue dans la production ou la reproduction des inégalités sexuées. Est-ce qu'elle fonctionne, ainsi que l'affirme M. Duru-Bellat, « [...] comme une caisse de résonance d'inégalités prévalant dans la société? » Serait-elle seulement « sexiste par abstention?» (Duru-Bellat, 2008, p. 147).

L'école dispose d'une certaine autonomie par rapport à la société dans laquelle elle est insérée. Néanmoins, elle n'est pas totalement imperméable aux phénomènes sociaux qui lui sont externes. Dans cette perspective, il importe, par rapport à notre questionnement, de faire le point sur les inégalités de pratiques physiques selon le sexe à l'extérieur de l'école. Prenant appui sur les enquêtes les plus récentes, C. Louveau (2004a) révèle les tendances suivantes pour la situation française: la pratique des activités physiques a augmenté dans des proportions non négligeables au cours des dernières décennies; ce développement concerne la pratique des hommes et des femmes mais les inégalités dans ce domaine perdurent; même si le nombre de pratiquantes a globalement augmenté, les femmes de milieux populaires, les inactives professionnellement, celles qui ont un faible niveau de diplôme et des revenus modestes pratiquent beaucoup moins que les autres.

Par ailleurs, les femmes ne pratiquent pas tout à fait les mêmes activités physiques que les hommes. Au cours d'un long processus historique, certaines pratiques se sont construites comme disciplines féminines (activités physiques artistiques, activités physiques d'entretien, etc.). D’autres se sont constituées comme activités physiques masculines (rugby, football, haltérophilie, boxe, etc.). Plutôt que d'employer le terme de féminisation des pratiques physiques, C. Louveau (2004b) propose d'employer la notion de sexuation. Ce qui rend compte aussi bien des aspects numériques (la proportion de pratiquantes) que des processus qualitatifs d'apparition et de transformation des pratiques. Pour cet auteur, la sexuation des pratiques physiques entretient un rapport d'homologie avec la sexuation du travail social et domestique. En outre, elle contribue puissamment à l'élaboration des modèles féminins et masculins.

Les travaux qui viennent d'être cités prennent appui sur des enquêtes réalisées généralement auprès de populations essentiellement adultes. Qu'observe-t-on pour les plus jeunes? Deux publications assez récentes permettent de faire le point (Ministère de la Jeunesse, des Sports et de la vie associative, 2004; Davisse, 2006). Ces travaux montrent, entre autres, que le processus de socialisation primaire 
prédispose différemment les filles et les garçons à la pratique des activités physiques. Et, de ce point de vue, nous relevons quatre principaux résultats : les filles, en règle générale, pratiquent moins fréquemment que les garçons (surtout dans les milieux populaires); elles ne pratiquent pas les mêmes activités (la distinction entre pratiques féminines et pratiques masculines est déjà très nette); elles ne sont pas aussi attirées que les garçons par les modalités de pratique compétitive; à partir d'un certain âge (notamment à l'adolescence), l'écart de pratique entre les filles et les garçons s'accroît.

Les travaux qui ont été évoqués jusqu'à présent à propos de la pratique des activités physiques concernent principalement ce qui se déroule en dehors de l'école. Comparativement, il existe très peu d'études centrées sur le sport scolaire. En France, le premier travail qui aborde le sujet est publié au début des années 1980 mais il n'intègre pas la question des inégalités de pratique selon le sexe (Chifflet, 1982). Pour cela, il faut attendre la recherche menée par M. Reneaud. Elle montre que la proportion de pratiquantes est comparativement plus forte dans les associations sportives scolaires que dans les structures extrascolaires. Apparemment le sport scolaire serait susceptible de jouer un rôle important dans la réduction des écarts de pratique selon le sexe. Mais, dans ce domaine, l'auteure demeure cependant prudente. Elle écrit « qu'en matière d'appropriation par les filles du fait culturel que constitue le sport, les associations sportives scolaires possèdent une avance incontestable sur l'organisation sportive fédérale, mais leur taux de féminisation ne saurait masquer la persistance d'inégalités » (Reneaud, 1988, p. 248). Une autre recherche publiée quatre ans plus tard corrobore ces résultats. Elle révèle que les écarts selon le sexe sont beaucoup moins marqués dans le cadre scolaire qu'à l'extérieur de celui-ci: 17,5\% des garçons et 12,3\% des filles pratiquent dans le cadre de l'association sportive de leur établissement; pour la pratique sportive en club en dehors de l'école, les proportions sont respectivement de 53,5\% et $29 \%$ (Combaz, 1992). Ces deux recherches montrent que la différence de pratique est moins prononcée au sein du sport scolaire que dans les structures extrascolaires. Néanmoins, les proportions de pratiquants y sont aussi bien plus faibles.

Ces premiers travaux ont ouvert des perspectives mais de nombreuses pistes restent à explorer. En premier lieu, il convient de prendre en considération simultanément les différents types de pratiques (sport scolaire, sport en club, activités physiques en dehors de toute structure institutionnelle, etc.). Loin d'être antinomiques, ces différentes modalités de pratique peuvent se cumuler et, à cet égard, il importe d'étudier les pratiques multiples (Augustini et al., 1996). Par ailleurs, il importe d'actualiser les résultats obtenus à la fin des années 1980. De ce point de vue, on peut se demander si la progression de la pratique féminine observée depuis quelques années a aussi concerné l'école. Le sport scolaire tel qu'il est organisé actuellement se différencie-t-il suffisamment des pratiques extrascolaires 
pour être en mesure de réduire les écarts habituellement observés entre les filles et les garçons ou reproduit-il ce que l'on constate à l'extérieur de l'école?

\section{Les différents types de pratique physique et sportive: éléments de définition}

L'une des faiblesses des deux recherches citées ci-dessus (Reneaud, op. cit. et Combaz, op. cit.) est de ne pas mettre suffisamment en perspective les différentes modalités de pratique des activités physiques. Remédier à cela suppose, au préalable, d'être en mesure de les distinguer clairement. Les données à notre disposition permettent d'identifier trois types de pratique: le sport scolaire; le sport en club à l'extérieur de l'école; la pratique dite « libre » en dehors de l'école. Les types de pratique développés dans ces trois secteurs ne sont pas identiques et nous faisons l'hypothèse qu'ils n'attirent pas nécessairement les filles et les garçons de la même manière. Ajoutons que ces trois types de pratique ne sont pas incompatibles. Ils peuvent se cumuler et donner lieu à lieu à un certain nombre de combinaisons qui n'ont pas forcément le même sens pour les pratiquants. Ainsi, un élève pourra souhaiter pratiquer un sport dans un club à l'extérieur de l'école pour bénéficier d'un entraînement régulier et pour participer à des compétitions. Il pourra aussi souhaiter, par ailleurs, s'adonner à des activités physiques de manière beaucoup plus informelle et de façon plus irrégulière. Tâchons de préciser ce qui distingue les trois formes de pratique retenues.

\section{Le sport scolaire}

Depuis la création des premières sociétés sportives scolaires à la fin du XIX siècle en France, le sport scolaire occupe une position ambivalente, à l'interface des valeurs éducatives de l'école et des enjeux de la confrontation sportive compétitive (Thibault, 1991). Du point de vue de l'affirmation de son identité, le sport scolaire a cherché dès les années 1960, à afficher sa spécificité en adaptant ses modalités d'organisation aux contraintes particulières du contexte de l'école afin de se distinguer du fonctionnement des clubs sportifs extrascolaires. Ainsi, dès 1966, certains enseignants d'EPS ont imaginé une organisation particulière pour mieux répondre à la diversité des attentes des élèves: les associations sportives dites de «masse ». À partir de 1972, cette formule originale a été officialisée et, depuis lors, deux modes de fonctionnement cohabitent au sein du sport scolaire: la forme traditionnelle de rencontres compétitives (calqué sur le modèle du club sportif extrascolaire) et la formule «masse » (appelée actuellement formule district). Les objectifs visés ne sont pas du tout identiques. L'organisation traditionnelle cherche essentiellement à faire émerger l'élite sportive. La formule « masse » se propose au contraire d'accueillir le plus grand nombre d'élèves quel que soit leur niveau de performance. Dans cette dernière perspective, les rencontres orga- 
nisées ne tiennent pas compte des critères habituels d'âge et de sexe. Ainsi, les filles et les garçons peuvent, par exemple, être amenés à évoluer ensemble au sein des mêmes équipes lorsqu'il s'agit de sports collectifs.

\section{Le sport en club en dehors de l'école}

Telles que nous les appréhendons ici, les pratiques développées dans ce cadrelà ont pour principal objectif la compétition. Celle-ci obéit à des règlements très précis et elle est organisée institutionnellement sous la forme de différentes fédérations qui structurent les rencontres du plus bas niveau (généralement le département) jusqu'à l'échelon international.

Le sport en club implique généralement la participation à des entraînements réguliers et l'apprentissage de gestes techniques très codifiés. Les statistiques montrent que les garçons et les hommes sont plus investis dans ce type de pratique que les filles et les femmes (cf. les travaux évoqués plus haut).

\section{La pratique libre en dehors de l'école}

Plusieurs dimensions la distinguent des deux autres types de pratique. Elle concerne les activités physiques qui se déroulent en dehors de toute structure institutionnelle. En règle générale, elle ne donne pas lieu à des rencontres compétitives (si ce n'est à titre amical et en ne respectant pas nécessairement les règlements officiels). Par ailleurs, elle se caractérise par une grande hétérogénéité de pratiques. Il peut s'agir de toutes les formes de pratiques urbaines: football joué au pied des immeubles dans les quartiers populaires, skateboard ou roller pratiqué dans les rues, etc. La pratique libre concerne également une grande partie des loisirs de pleine nature (randonnée pédestre, sports d'hiver, sports aquatiques, etc.). Elle recouvre aussi la plupart des activités physiques dites d'entretien (jogging dominical, exercices physiques réalisés au domicile, etc.). La dernière enquête nationale sur les pratiques physiques des Français montre qu'en l'espace de quelques années, la pratique libre s'est considérablement développée. En outre, les différences de pratique selon le sexe y sont moins marquées qu'au sein des pratiques institutionnalisées (Ministère de la jeunesse et des sports et de la vie associative, 2005).

\section{La méthodologie}

Du point de vue empirique, nous avons eu l'opportunité de réaliser l'analyse secondaire d'une enquête nationale menée par les services statistiques du ministère de l'Éducation nationale (DEPP) ${ }^{(3)}$ au cours de l'année 2006. L'un des intérêts de ce type de démarche est qu'elle permet généralement de travailler sur des

(3). Direction de l'évaluation, de la prospective et de la performance. 
enquêtes extensives réalisées à l'échelon national ou international. Ce qui permet l'accès à un certain degré de généralisation des résultats obtenus. La qualité de l'analyse secondaire dépend en partie des informations qui accompagnent les données transmises. À cet égard, nous avons eu accès au fichier de données, aux procédures de codage et au questionnaire utilisé.

L'enquête que nous avons eu l'occasion de réexploiter a permis d'interroger, à partir d'un échantillonnage national, 1954 élèves. La base de sondage choisie est constituée des collèges, des lycées généraux et professionnels, publics et privés de la métropole et des départements d'Outre-mer. La méthode d'échantillonnage utilisée est le sondage stratifié. Trois critères ont été retenus pour réaliser cette stratification: la taille des établissements, le fait qu'ils soient classés ou non en zone d'éducation prioritaire (ZEP), le fait qu'ils soient implantés en zone urbaine ou en zone rurale. Ainsi, 360 collèges, 192 lycées généraux et 177 lycées professionnels ont été tirés au sort. Dans chaque établissement, un ou plusieurs élèves ont été tirés au sort. Le nombre d'élèves tirés au sort varie de 1 à 3 en fonction de la taille de l'établissement. Les taux de réponse sont plutôt élevés: 89 \% (ils sont un peu plus élevés en collège qu'en lycées). La représentativité des répondants appréciée selon les trois critères de stratification a été jugée très satisfaisante (cf. le rapport de J. Benhaim-Grosse, 2007).

Signalons que lors du transfert des données par la DEPP, un certain nombre de variables (sociologiquement pertinentes) ne nous ont pas été communiquées: appartenance ou non des établissements à une ZEP, implantation de ceux-ci en zone urbaine ou zone rurale. Par ailleurs, aucune variable ne permet d'identifier précisément l'origine sociale des élèves. Ceci constitue une limite à notre travail compte tenu de l'importance de cette variable dans la différenciation sociale des pratiques sportives. Pour tenir compte de cela, l'origine sociale a été appréhendée ici, de manière indirecte, par le type d'établissement dans lequel l'élève est scolarisé: collège, lycée ou lycée professionnel. Comparativement, les collèges et les lycées scolarisent moins d'élèves de milieux modestes que les lycées professionnels (cf. tableau Al en annexe).

L'enquête permet de mettre en perspective les trois types de pratique physique distingués et d'apprécier la participation des filles et des garçons selon l'âge et l'établissement scolaire. Par ailleurs, elle offre la possibilité d'identifier la nature des activités physiques pratiquées par les élèves.

\section{Résultats}

\section{La pratique des activités physiques: quelles différences entre filles et garçons?}

La pratique des activités physiques a été appréhendée à travers huit modalités qui recouvrent toutes les combinaisons envisageables: allant du cumul des trois 
types de pratique (sport scolaire, sport en club en dehors de l'école et pratique libre) jusqu'à l'absence totale de pratique. Les résultats consignés dans le tableau 1 révèlent trois principales tendances. Les filles sont quasiment trois fois plus nombreuses que les garçons à ne pratiquer aucune activité physique. Les garçons sont davantage représentés que les filles parmi ceux qui pratiquent en club et librement et parmi ceux qui cumulent les trois types de pratique. En définitive, on observe qu'en association ou non avec les deux autres types de pratique, le sport scolaire ne se distingue pas particulièrement quant à sa capacité éventuelle à réduire les inégalités selon le sexe (sauf à considérer les résultats relatifs aux élèves qui pratiquent uniquement dans le cadre du sport scolaire: les filles sont deux fois plus nombreuses que les garçons mais cela ne concerne qu'une très faible proportion d'élèves).

Lorsqu'on prend en compte simultanément le sexe, l'âge, l'établissement scolaire fréquenté et les différentes modalités de pratique grâce à l'analyse factorielle des correspondances multiples, les résultats obtenus ne sont guère différents de ceux qui viennent d'être présentés. Le premier plan factoriel oppose nettement trois modalités de pratique pour lesquelles les filles et les garçons ne se positionnent pas de la même manière (graphique 1).

L'usage conjoint de la statistique descriptive et de l'analyse multidimensionnelle montre que les filles de lycée professionnel âgées de 16 à 18 ans sont les plus nombreuses à ne pratiquer aucune activité physique (tableau A2 en annexe) Celles qui sont âgées de 19 ans et plus ainsi que leurs camarades garçons âgés de 16 ans et moins et de 19 ans et plus sont les plus représentés parmi ceux qui s'adonnent uniquement à la pratique libre.

Tableau I. La pratique des activités physiques et sportives selon le sexe des élèves (pourcentages et écarts à l'indépendance)

\begin{tabular}{|l|r|r|r|}
\hline Sport scolaire + club + pratique libre & \multicolumn{1}{|c|}{ Filles } & \multicolumn{1}{c|}{ Garçons } & Ensemble \\
\hline Sport scolaire + pratique libre & $8,9(-20,8)$ & $\mathbf{1 3 , 3}(\mathbf{2 0 , 8 )}$ & 11,1 \\
\hline Sport scolaire + club & $4,7(-0,8)$ & $4,9(0,8)$ & 4,8 \\
\hline Sport scolaire uniquement & $1,9(-2,7)$ & $2,5(2,7)$ & 2,2 \\
\hline Club + pratique libre & $2,4(5,3)$ & $1,2(5,3)$ & 1,8 \\
\hline Pratique libre uniquement & $23,2(-69,4)$ & $\mathbf{3 8}(\mathbf{6 9}, \mathbf{4})$ & 30,7 \\
\hline Club uniquement & $22,8(-1,1)$ & $23(1,1)$ & 22,9 \\
\hline Aucune pratique & $9,3(6,8)$ & $7,9(-6,8)$ & 8,6 \\
\hline Toutes modalités confondues & $\mathbf{2 6 , 8 ( 8 2 , 7 )}$ & $9,2(-82,7)$ & 17,9 \\
\hline
\end{tabular}


Le khi-deux est significatif à un seuil <.001. Pour repérer les cases du tableau qui révèlent les différences les plus saillantes, nous avons mentionné entre parenthèses les écarts à l'indépendance. Lorsqu'ils sont en italiques, ils révèlent une sous-représentation; lorsqu'ils sont en caractère gras, ils indiquent une sur représentation.

Les collégiens et les collégiennes âgés de 12 ans et moins se distinguent des autres élèves en étant proportionnellement les plus nombreux parmi ceux qui cumulent les trois types de pratique (les collégiens de 15 ans et plus y sont également bien représentés. Cf. tableau A2 en annexe).

Le deuxième plan factoriel $(3-4)$ permet de faire émerger, outre ce que nous avons déjà repéré avec le premier, la modalité pratique en club uniquement (graphique Al en annexe). Sont prioritairement associés à ce type de pratique: les collégiennes de 13 ans, les lycéennes de 15 ans et plus et les lycéens de 17 ans.

Le troisième plan factoriel (plan 5 - 6) met en évidence les modalités suivantes: club et sport scolaire, pratique libre et sport scolaire, sport scolaire seul (graphique A2 en annexe). La première concerne essentiellement les garçons (collégiens âgés de 12 ans et moins, lycéens âgés de 17 ans et plus). À la seconde sont associés principalement les collégiens et les collégiennes de 12 ans et moins et les collégiens de 14 ans. Les élèves qui pratiquent uniquement dans le cadre du sport scolaire sont extrêmement minoritaires (moins de $2 \%$ de l'ensemble). Parmi ceux-ci, les filles de lycée professionnel les plus âgées (18 ans et plus) se distinguent des autres ainsi que les collégiens les plus jeunes.

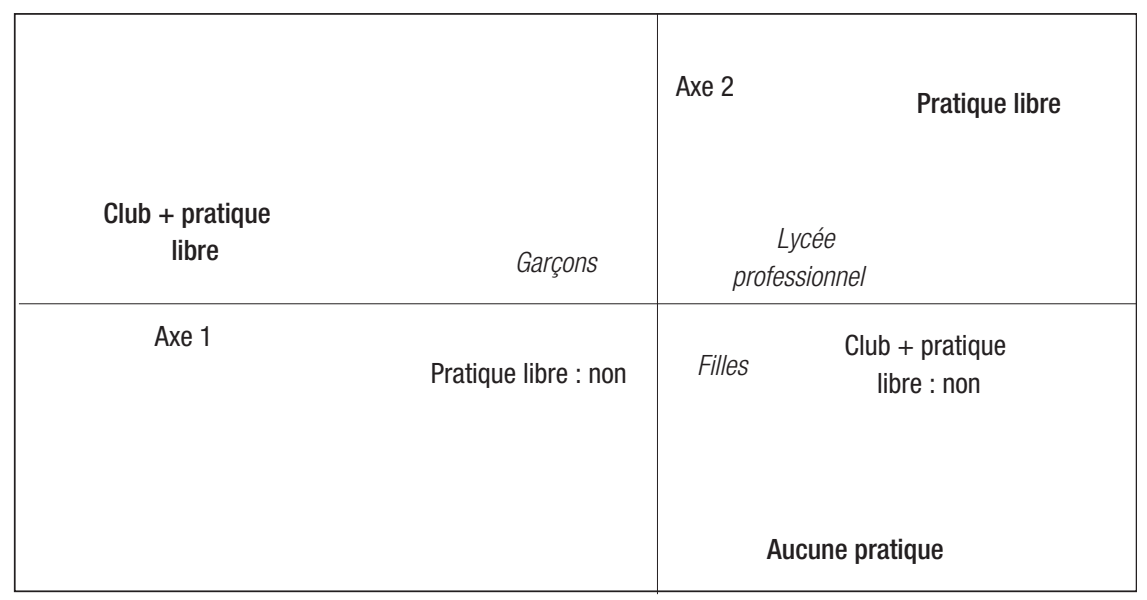

Graphique I- La pratique des activités physiques et sportives. Analyse factorielle des correspondances multiples (plan I-2)

Pour le dépouillement et l'interprétation des résultats issus des analyses factorielles mises en œuvre dans cette recherche, nous avons pris appui sur les ouvrages 
suivants: Escofier, Pagès (1998); Jambu (1989); Lebart, Morineau, Piron (2000). Les modalités de pratique qui contribuent le plus à la construction des axes sont présentées en caractères gras. Elles ont ici, sur ce graphique, une contribution quatre à cinq fois plus élevée que la contribution moyenne. Le sexe, l'établissement fréquenté et l'âge ont été introduits dans l'analyse en tant que modalités illustratives (en caractères italiques). Nous avons retenu uniquement celles qui sont les plus corrélées aux axes. Pour sélectionner ces dernières, nous avons repris les indications fournies par Lebart, Morineau, Piron (valeurs-test supérieures ou égales à 2).

L'analyse détaillée qui vient d'être menée ne remet pas fondamentalement en cause les résultats déjà observés dans le tableau 1 : le sport scolaire ne se différencie pas véritablement des autres modalités de pratique du point de vue des inégalités selon le sexe. Ceci semble accréditer la thèse défendue par M. DuruBellat à propos du rôle que joue l'école dans la reproduction des inégalités sexuées. Mais peut-on vraiment exonérer l'institution scolaire de toute responsabilité dans la constitution de celles-ci? Depuis la moitié des années 1970, de nombreux travaux conduits dans le champ de la sociologie de l'éducation, ont montré que si les inégalités scolaires résultent essentiellement de facteurs externes à l'école (inégalités sociales, culturelles, économiques, entre les sexes, etc.), le fonctionnement « interne » du système éducatif contribue, pour une part, à leur « fabrication » (effet maître, effet établissement, nature des contenus enseignés, etc.). Dans cette perspective, on peut se demander dans quelle mesure le sport scolaire participe à la constitution de ces inégalités ou s'il n'est que le simple miroir de ce qui se passe en dehors de l'école du point de vue de la pratique des activités physiques. Répondre à cette question suppose d'avoir des données permettant d'analyser précisément les pratiques mises en œuvre par les enseignants d'EPS en charge du sport scolaire dans les établissements. Lapproche macrosociologique privilégiée ici ne correspond pas à cette orientation. Néanmoins, nous disposons d'une information qui n'est pas nécessairement négligeable au sujet du rôle que le sport scolaire peut jouer dans la constitution des inégalités entre les sexes: il s'agit de la nature des activités physiques pratiquées. Les travaux sociologiques centrés sur les inégalités sexuées en EPS ont montré l'importance de prendre en considération cette dimension. Quel effet peut-elle avoir pour le sport scolaire? Les recherches menées dans le champ de la sociologie du sport montrent que les aspirations des filles et des garçons vis-à-vis des pratiques physiques diffèrent assez nettement. Certaines activités sont essentiellement pratiquées par les filles et d'autres sont plutôt l'apanage des garçons (sachant que la socialisation primaire joue un rôle décisif dans l'élaboration de ces « choix »). De quelle manière le sport scolaire prend-il en considération ces goûts sportifs? Les activités auxquelles s'adonnent les élèves en son sein correspondent-elles de manière comparable aux souhaits des filles et des garçons ou observe-t-on un déséquilibre à ce sujet? 
Les activités physiques pratiquées dans le cadre du sport peuvent-elles être la source d'inégalités entre filles et garçons?

Formellement, l'éventail des activités physiques susceptibles d'être pratiquées dans le cadre du sport scolaire n'est pas fondamentalement différent de ce qui existe en dehors de l'école. Qu'en est-il en réalité? Les résultats consignés dans le tableau 2 révèlent que certaines activités prédominent (badminton, football, handball, volley-ball, athlétisme et basket-ball). Comparativement, les autres activités sont moins souvent pratiquées (activités physiques d'expression, activités physiques de pleine nature, sports de combat, activités physiques d'entretien, etc.).

Tableau 2. Activités physiques pratiquées par les élèves dans le cadre du sport scolaire (en \%)

\begin{tabular}{|c|c|c|c|c|}
\hline Activités physiques pratiquées & Garçons & Filles & Ensemble & $\begin{array}{c}\text { Différences } \\
\text { garçons-filles }\end{array}$ \\
\hline Badminton & 23,7 & 21,5 & 22,7 & $2,2 \mathrm{~ns}$ \\
\hline Football & 37,2 & 2,3 & 21,7 & 34,9 ** \\
\hline Handball & 21,9 & 21,5 & 21,7 & $0,4 \mathrm{~ns}$ \\
\hline Volley-ball & 14,4 & 15,7 & 15,0 & $-1,3 \mathrm{~ns}$ \\
\hline Athlétisme & 11,2 & 19,2 & 14,7 & $-8,0$ * \\
\hline Basket-ball & 11,6 & 13,4 & 12,4 & $-1,8 \mathrm{~ns}$ \\
\hline Sports de raquette ${ }^{(1)}$ sauf badminton & 12,6 & 5,8 & 9,6 & $6,8^{*}$ \\
\hline APPN (2) autres que l'escalade & 7,0 & 9,3 & 8,0 & $-2,3$ ns \\
\hline Escalade & 3,3 & 9,9 & 6,2 & $-6,6^{\star \star}$ \\
\hline Danses et autres APEX ${ }^{(3)}$ & 2,8 & 9,9 & 5,9 & $-7,1^{\star *}$ \\
\hline Divers ${ }^{(4)}$ & 7,0 & 4,7 & 5,9 & $2,3 \mathrm{~ns}$ \\
\hline Musculation & 4,7 & 5,8 & 5,2 & $-1,1 \mathrm{~ns}$ \\
\hline Aérobic, step, stretching & 1,4 & 8,1 & 4,4 & $-6,7^{\star *}$ \\
\hline Natation sportive & 1,9 & 6,4 & 3,9 & $-4,5^{*}$ \\
\hline Gymnastique volontaire & 2,8 & 5,2 & 3,9 & $-2,4 \mathrm{~ns}$ \\
\hline Sports de combat (judo, lutte et boxe) & 2,3 & 3,5 & 2,8 & $-1,2 \mathrm{~ns}$ \\
\hline Rugby & 4,2 & 0,6 & 2,6 & $3,6^{*}$ \\
\hline
\end{tabular}

(1) Cette appellation renvoie aux activités suivantes: tennis, squash et tennis de table.

(2) Il s'agit des activités physiques de pleine nature autres que l'escalade (voile, canoë-kayak, planche à voile, etc.).

(3) Il s'agit des activités physiques d'expression autres que la danse (natation synchronisée, gymnastique rythmique et sportive, arts du cirque, etc.).

(4) Ont été classées dans cette rubrique Divers les activités physiques pratiquées par moins de $1 \%$ des élèves.

** Différences significatives à un seuil inférieur ou égal à $1 \%$ 
* Différences significatives à un seuil inférieur ou égal à $5 \%$

ns: différences non significatives statistiquement

Cette répartition des activités pratiquées s'avère en net décalage avec les aspirations des élèves. Nous avons comparé les souhaits des élèves pour l'ensemble de l'échantillon ( $\mathrm{N}=1954)$ et pour ceux qui participent au sport scolaire ( $\mathrm{N}$ = 387). Les résultats convergent largement (tableau A3 en annexe). Ils montrent que le décalage entre les pratiques et les souhaits concerne aussi bien les filles que les garçons. Les activités physiques d'expression (danse, natation synchronisée, etc.), les activités physiques d'entretien (aérobic, step, etc.) et la gymnastique sportive sont largement plébiscitées par les filles. Elles sont relativement peu souvent pratiquées dans le cadre du sport scolaire. Les activités physiques de pleine nature suscitent également un fort engouement des élèves (aussi bien des filles que des garçons). Elles sont assez peu souvent pratiquées dans le cadre du sport scolaire. Seuls le football et, dans une moindre mesure, le handball semblent faire exception: ils font partie des activités le plus souvent pratiquées dans le cadre du sport scolaire et elles correspondent assez bien aux aspirations des garçons. Mais ceci ne nous permet pas d'affirmer que la répartition des activités physiques pratiquées constitue une source importante d'inégalités entre les sexes.

Face aux analyses qui viennent d'être développées, on pourra objecter que certaines activités physiques souvent pratiquées au sein du sport scolaire s'adressent, a priori, aussi bien aux filles qu'aux garçons parce qu'elles ne sont pas spécifiquement «féminines » ou particulièrement « masculines ». C'est le cas notamment $\mathrm{du}$ badminton et du volley-ball. Néanmoins, la question du décalage entre les aspirations et les pratiques demeure: ces deux activités ne sont pas particulièrement prisées des élèves. Ceci soulève une autre objection. Jusqu'à quel point le sport scolaire doit-il tenir compte des souhaits des élèves? En prenant largement en considération leurs aspirations ne risque-t-on pas de reproduire les stéréotypes sexués les plus traditionnels avec, d'un côté des filles pratiquant surtout des activités dites « féminines » et, de l'autre, des garçons s'adonnant plutôt à la pratique d'activités classiquement considérées comme " masculines »? Répondre à cette question suppose que l'on puisse interroger les plus hauts responsables du sport scolaire pour être en mesure d'analyser précisément les finalités qui sont visées, en particulier, celles qui concernent les inégalités de pratique entre garçons et filles.

\section{Conclusion}

Nous avons montré que les inégalités de pratiques physiques selon le sexe observé au sein de l'école sont assez comparables à celles que l'on relève à l'extérieur. Ce résultat paraît aller à l'encontre des tendances mises en évidence par la littérature existante. La divergence provient essentiellement des modes de calcul 
utilisés. Dans les travaux de M. Reneaud (op. cit.) et de G. Combaz (op. cit.), les différentes modalités de pratique ne sont pas prises en considération simultanément. Ce qui permet de montrer que les inégalités de pratique selon le sexe sont plus réduites au sein du sport qu'à l'extérieur de l'école. Mais, en réalité, les élèves qui pratiquent dans le cadre du sport scolaire cumulent divers types de pratique (sport en club en dehors de l'école et pratique libre). Pour l'heure, le sport scolaire ne semble pas véritablement en mesure d'attirer des filles et des garçons qui ne pratiquent aucune activité physique en dehors de l'école (ils représentent moins de $2 \%$ dans notre échantillon).

Ceci étant posé, le résultat que nous avons mis en évidence ne nous permet nullement d'affirmer que l'école se contente seulement de reproduire des inégalités qui existent en dehors d'elle. L'approche macrosociologique que nous avons privilégiée dans cet article gomme totalement des différences de fonctionnement qui peuvent exister localement dans tel ou tel établissement. Des écrits militants laissent entendre que certaines pratiques développées dans des établissements classés en éducation prioritaire sont susceptibles d'attirer des filles de milieux populaires qui ne pratiquent aucune activité physique en dehors de l'école (Sels, 2003). Dans cette perspective, il importe d'entreprendre, à présent, des investigations d'ordre plus microsociologique pour vérifier dans quelle mesure les formes d'organisation proposées localement dans les établissements scolaires peuvent (ou non) encourager l'investissement aussi bien des filles que des garçons. À ce titre, on peut supposer, par exemple, qu'une complémentarité judicieuse entre le secteur traditionnel et la formule district peut susciter un engagement plus marqué des élèves (quel que soit leur sexe). Par ailleurs, il convient sans doute de voir de quelle manière les orientations impulsées par les instances nationales peuvent être relayées localement (aussi bien à l'échelon académique ou départemental qu'au niveau de l'établissement scolaire).

Gilles Combaz, laboratoire éducation, cultures et politiques, université Lyon 2 (EA 4571).

Olivier Hoibian, laboratoire Sports, organisations, identités, université de Toulouse 3 (EA 3690).

\section{BIBLIOGRAPHIE}

Auduc J.-L. (2009). Sauvons les garçons! Paris: éditions Descartes \& Cie.

Augustini M., Irlinger P., Louveau C. (1996). Un aspect négligé par l'étude de l'engagement sportif: la multipratique et ses modalités. Loisir et société, vol. 19, n 1, p. 237-262.

Benhaim-Grosse J. (2007). Image du sport scolaire et pratiques d'enseignement au collège et au lycée. Paris: DEPP.

Brugeilles C., Cromer S., Panissal N. (2009). Le sexisme au programme. Représentations sexuées dans les lectures de référence à l'école. Travail, genre et sociétés, n² 21, p. 107-129. 
Chifflet P. (1982). Les lycéens et l'association sportive. STAPS, n 6, p. 36-46.

Cogérino G. (2005). Filles et garçons en EPS. Paris: éditions EPS.

Combaz G. (1992). L’association sportive scolaire: un moyen pour réduire les inégalités d'accès à la pratique des activités physiques? Science et motricité, n 18, p. 45-52.

Combaz G., Hoibian O. (2008). Le rôle de l'école dans la construction des inégalités de sexe. L'exemple de l'éducation physique et sportive. Travail, genre et sociétés, n 20, p. 129-150.

Davisse A. (2006). Filles et garçons dans les activités physiques et sportives: de grands changements et de fortes permanences. In A. Dafflon-Novelle (éd.) Filles-garçons. Socialisation différenciée? Grenoble: PUG, p. 287-301.

Duru-Bellat M. (1995). Filles et garçons à l'école, approches sociologiques et psychosociales. La construction scolaire des différences entre les sexes. Revue française de pédagogie, n 110, p. 75-109.

Duru-Bellat M. (2002). Les inégalités sociales à lécole: genèse et mythes. Paris: PUF.

Duru-Bellat M. (2008). La reproduction des rapports sociaux de sexe: quelle place pour l'institution scolaire? Travail, genre et sociétés, n 19, p. 131-149.

Escofier B., Pagès J. (1998). Analyses factorielles simples et multiples. Objectifs, méthodes et interprétation. Paris: Dunod.

Jambu M. (1989). Exploration informatique et statistique des données. Paris: Dunod.

Lebart L., Morineau A., Piron M. (2000). Statistique exploratoire multidimensionnelle. Paris: Dunod.

Lemarchant C. (2007). La mixité inachevée. Garçons et filles minoritaires dans les filières techniques. Travail, genre et sociétés, nº 18, p. 47-64.

Louveau C., Davisse A. (1998). Sports, école, société: la différenciation des sexes. Féminin, masculin et activités sportives. Paris: éditions L'Harmattan.

Louveau C. (2004a). Pratiquer une activité physique ou sportive: persistance des inégalités parmi les femmes. Recherches féministes, vol. 17, nº 1, p. 39-76.

Louveau C. (2004b). Sexuation du travail sportif et construction sociale de la féminité. Cahiers du genre, n³6, p. 163-183.

Ministère de l'Éducation nationale (2006). Égalité des filles et des garçons?

www.education.gouv.fr, (consultation: octobre 2010).

Ministère de l'Éducation nationale (2010). Filles et garçons sur le chemin de l'égalité, de l'école à l'enseignement supérieur. www.education.gouv.fr, (consultation: avril 2010).

Ministère de la jeunesse et des sports et de la vie associative (2004). Les adolescents et le sport. Paris: INSEP.

Ministère de la Jeunesse et des sports et de la vie associative (2005). La pratique des activités physiques et sportives en France. Paris: INSEP.

Mosconi N. (2001). Comment les pratiques enseignantes fabriquent-elles de l'inégalité entre les sexes? Les dossiers des sciences de l'éducation, n 5, p. 97-109.

Petrovic C. (2004). Filles et garçons en éducation: les recherches récentes. Carrefours de l'éducation, $\mathrm{n}^{\circ} 17$, p. $76-100, \mathrm{n}^{\circ} 18$, p. 146-175.

Reneaud M. (1988). L’association sportive et la réduction des inégalités. Un analyseur du sport féminin. In P. Chifflet (éd.), Méthodologie et didactique de l'éducation physique et sportive. Paris : éditions AFRAPS, p. 245-253. 
Rosenwald F. (2006). Les filles et les garçons dans le système éducatif. Note d’information, n 06.06 , p. 1-6.

Sels J. (2003). Les pratiques sportives des filles à l'UNSS. Contre-pied, n 12, p. 17-20.

Thibault J. (1991). Sports et éducation physique, 1870-1970. Paris: éditions Vrin.

Trottin B., Cogérino G. (2009). Filles et garçons en EPS: approche descriptive des interactions verbales entre enseignants et élèves. STAPS, nº 83, p. 69-85.

Vigneron C. (2006). Les inégalités de réussite en EPS entre filles et garçons: déterminismes biologiques ou fabrication scolaire? Revue française de pédagogie, n 154, p. 111-124.

Vouillot F. (2007). L'orientation aux prises avec le genre. Travail, genre et sociétés, n 18, p. 87-108.

\section{ANNEXES}

\section{Tableau AI. L'origine socioprofessionnelle des élèves du second degré en 2006}

\begin{tabular}{|l|r|r|r|}
\hline \multicolumn{1}{|c|}{ PCS } & \multicolumn{2}{|c|}{ Lycées } & $\begin{array}{c}\text { Lycées profession- } \\
\text { nels }\end{array}$ \\
\hline $\begin{array}{l}\text { Agriculteurs exploi- } \\
\text { tants }\end{array}$ & 2,3 & 2,2 & 1,6 \\
\hline $\begin{array}{l}\text { Artisans et commer- } \\
\text { çants }\end{array}$ & 9,2 & 9,5 & 7,9 \\
\hline $\begin{array}{l}\text { Professions libérales } \\
\text { et cadres supérieurs }\end{array}$ & 15,9 & 23,3 & 5,8 \\
\hline $\begin{array}{l}\text { Professions intermé- } \\
\text { diaires }\end{array}$ & 13,9 & 16,9 & 12,0 \\
\hline Enseignants * & 3,3 & 5,2 & 1,0 \\
\hline Employés & 17,4 & 16,9 & 19,1 \\
\hline Ouvriers & 26,4 & 17,9 & 34,7 \\
\hline Autres ** & 11,6 & 8,1 & 17,9 \\
\hline
\end{tabular}

* Il s'agit des professeurs du second degré, des instituteurs et des professeurs des écoles.

** Cette catégorie comprend les retraités et les chômeurs n'ayant jamais travaillé.

Source: MEN, Repères et références statistiques sur les enseignements, la formation et la recherche, 2007, p. 105. 
Graphique Al - La pratique des activités physiques et sportives. Analyse factorielle des correspondances multiples (plan 3-4)

\begin{tabular}{|cc|cc|}
\hline & & Axe 4 & \\
& & & \\
& & & \\
& Filles & Collège & oui \\
Aucune activité & Lycée professionnel & Garçons & Axe 3 \\
& & & Trois pratiques \\
& & & cumulées \\
\hline
\end{tabular}

Graphique A2 - La pratique des activités physiques et sportives. Analyse factorielle des correspondances multiples (plan $5-6$ )

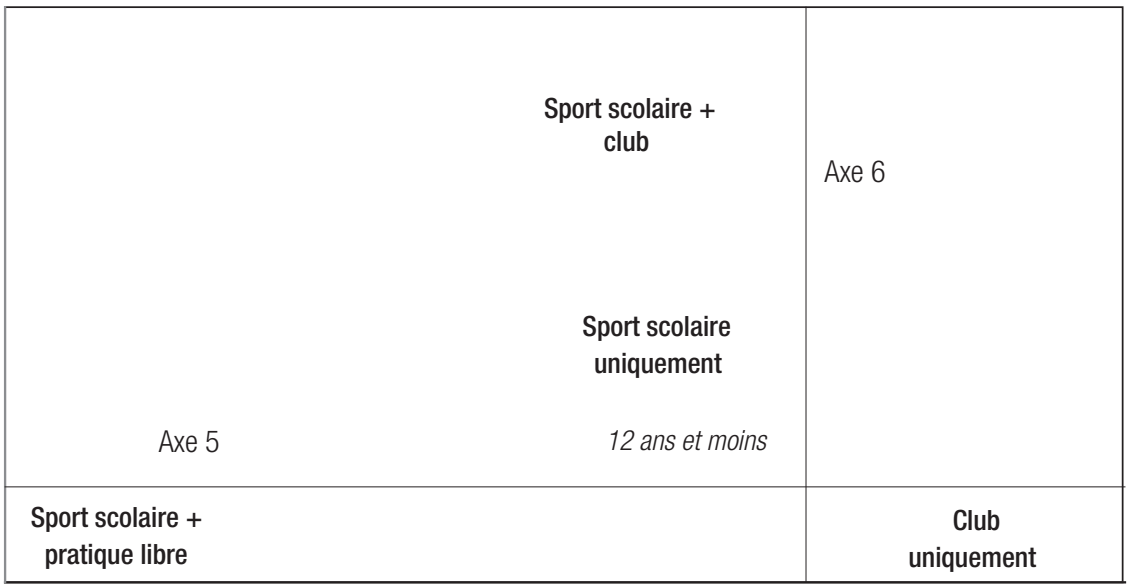

Tableau A2 - Les pratiques sportives des élèves: les trois pourcentages les plus élevés pour chacune des modalités*

Cumul des trois types de pratique (11,1\% sur l'ensemble)

Garçons de collège âgés de 12 ans et moins: 24,7 \%

Filles de collège âgées de 12 ans et moins : 20,6 \%

Garçons de collège âgés de 15 ans et plus: 17 \% 


\section{Club et pratique libre $(30,7 \%$ sur l'ensemble)}

Garçons de lycée âgés de 16 ans : $50 \%$

Garçons de collège âgés de 13 ans : 45,3 \%

Garçons de collège âgés de 15 ans et plus : 40,4 \%

\section{Sport scolaire et pratique libre ( $4,8 \%$ sur l'ensemble)}

Garçons de collège âgés de 12 ans et moins : 7,4 \%

Filles de collège âgées de 12 ans et moins : 7,4 \% Garçons de collège âgés de 14 ans: 7,3 \%

\section{Pratique libre uniquement (22,9\% sur l'ensemble)}

Filles de lycée professionnel âgées de 19 ans et plus : 38,2 \%

Garçons de lycée professionnel âgés de 19 ans et plus : 33,6 \% Garçons de lycée professionnel âgés de 16 ans et moins : 32,9\%

\section{Sport scolaire et club $(2,2 \%$ sur l'ensemble)}

Garçons de collège âgés de 12 ans et moins : 6, 2 \% Garçons de lycée âgés de 18 ans et plus: $5,1 \%$

Garçons de lycée âgés de 17 ans : 4,9\%

Club uniquement ( $8,6 \%$ sur l'ensemble)

Filles de collège âgées de 13 ans: 14,9\%

Filles de lycée âgées de 15 ans et plus: 12,2 Garçons de lycée âgés de 17 ans: 12,2 \%

\section{Sport scolaire uniquement (1,8 \% sur l'ensemble)}

Filles de lycée professionnel âgées de 18 ans : 7,4 \%

Garçons de collège âgés de 12 ans et moins : 6,2 \% Filles de lycée professionnel âgées de 19 ans et plus: $3,9 \%$

\section{Aucune activité physique (17,9\% sur l'ensemble)}

Filles de lycée professionnel âgées de 16 ans et moins : 52,9\%

Filles de lycée professionnel âgées de 17 ans : $45 \%$

Filles de lycée professionnel âgées de 18 ans : 42,6 \%

* Ces résultats sont issus du tableau qui croise les différentes modalités de pratique avec le sexe, l'âge et le type d'établissement scolaire fréquenté. 
Tableau A3 - La pratique des activités physiques et sportives: les souhaits des élèves. Résultats pour l'ensemble de l'échantillon $(N=1954)$ et pour ceux qui participent au sport scolaire $(N=387)$

\begin{tabular}{|c|c|c|c|c|c|c|}
\hline \multirow[t]{2}{*}{ Activités physiques } & \multicolumn{2}{|c|}{$\begin{array}{c}\text { Pratique } \\
\text { souhaitée (tout } \\
\text { l'échantillon) }\end{array}$} & \multirow[t]{2}{*}{$\begin{array}{l}\text { Différences } \\
\text { entre garçons } \\
\text { et filles }\end{array}$} & \multicolumn{2}{|c|}{$\begin{array}{c}\text { Pratique souhai- } \\
\text { tée (inscrits au } \\
\text { sport scolaire) }\end{array}$} & \multirow[t]{2}{*}{$\begin{array}{c}\text { Différences } \\
\text { entre garçons } \\
\text { et filles }\end{array}$} \\
\hline & $\begin{array}{l}\text { Gar- } \\
\text { çons }\end{array}$ & Filles & & $\begin{array}{l}\text { Gar- } \\
\text { çons }\end{array}$ & Filles & \\
\hline Danse & 5,2 & 22,2 & $17,0^{* *}$ & 8,8 & 26,7 & $17,9^{* *}$ \\
\hline Natation synchronisée & 5,5 & 15,6 & $10,1^{* *}$ & 6,5 & 23,8 & $\__{17,3^{\star *}}$ \\
\hline Aérobic, step, streching & 7,0 & 16,0 & $9,0^{\star \star}$ & 13,5 & 20,9 & \\
\hline Arts du cirque & 7,9 & 15,8 & $7,9^{\star \star}$ & 8,4 & 20,9 & $\__{12,5^{* *}}$ \\
\hline GRS & 2,7 & 10,3 & $7,6^{\star \star}$ & 4,0 & 16,9 & $12,9^{* *}$ \\
\hline Gymnastique sportive & 6,8 & 11,8 & $5,0^{\star *}$ & 9,3 & 18,0 & $-8,7^{*}$ \\
\hline Hip-hop & 23,4 & 26,9 & $-3,5 \mathrm{~ns}$ & 25,6 & 32,6 & $-7,0 \mathrm{~ns}$ \\
\hline Acrosport & 7,3 & 10,1 & $2,8^{*}$ & 11,6 & 15,1 & $-3,5 n s$ \\
\hline Roller & 19,7 & 19,4 & $0,3 \mathrm{~ns}$ & 24,7 & 27,9 & $-3,2 n s$ \\
\hline Badminton & 20,3 & 18,2 & $2,1 \mathrm{~ns}$ & 24,2 & 25,0 & $-0,8 \mathrm{~ns}$ \\
\hline Escalade (sur mur) & 23,2 & 20,4 & $2,8 \mathrm{~ns}$ & 25,6 & 30,2 & $-4,6 n s$ \\
\hline Judo & 14,3 & 11,5 & $2,8 \mathrm{~ns}$ & 14,9 & 14,0 & $0,9 \mathrm{~ns}$ \\
\hline Athlétisme & 12,4 & 8,5 & $3,9^{\star \star}$ & 15,3 & 14,0 & $1,3 \mathrm{~ns}$ \\
\hline Voile & 21,1 & 17,2 & $3,9^{*}$ & 27,4 & 28,5 & $-1,1 \mathrm{~ns}$ \\
\hline Volley-ball & 18,7 & 14,4 & $4,3^{*}$ & 24,7 & 19,8 & $4,9 \mathrm{~ns}$ \\
\hline Planche à voile & 27,5 & 23,0 & $4,5^{\star}$ & 33,5 & 34,9 & $-1,4 \mathrm{~ns}$ \\
\hline Escalade (site naturel) & 34,5 & 29,9 & $4,6^{*}$ & 38,6 & 43,6 & $-5,0 \mathrm{~ns}$ \\
\hline Escrime & 23,1 & 18,3 & $4,8^{\star \star}$ & 32,1 & 25,0 & $7,1 \mathrm{~ns}$ \\
\hline Natation sportive & 21,1 & 16,1 & $5,0^{\star \star}$ & 26,0 & 27,9 & $-1,9 \mathrm{~ns}$ \\
\hline Canoë-kayak & 36,0 & 30,9 & $5,1^{*}$ & 47,0 & 45,9 & $1,1 \mathrm{~ns}$ \\
\hline Ultimate & 14,1 & 9,0 & $5,1^{\star \star}$ & 21,4 & 15,1 & $6,3 \mathrm{~ns}$ \\
\hline Basket-ball & 19,6 & 14,2 & $5,4^{\star \star}$ & 20,9 & 19,2 & $1,7 \mathrm{~ns}$ \\
\hline Skate-board & 15,8 & 9,9 & $5,9^{* *}$ & 15,8 & 12,2 & $3,6 \mathrm{~ns}$ \\
\hline Lutte & 13,8 & 7,5 & $6,3^{\star \star}$ & 14,4 & 8,7 & $5,7 \mathrm{~ns}$ \\
\hline Alpinisme & 21,8 & 15,5 & $6,3^{\star \star}$ & 20,9 & 23,8 & $-2,9 \mathrm{~ns}$ \\
\hline Ski nordique & 18,2 & 11,5 & $6,7^{\star \star}$ & 22,8 & 15,1 & $7,7 \mathrm{~ns}$ \\
\hline Parapente & 33,2 & 26,1 & $7,1^{\star *}$ & 39,5 & 39,0 & $0,5 \mathrm{~ns}$ \\
\hline Boxe & 29,9 & 22,7 & $7,2^{* *}$ & 34,4 & 27,9 & $6,5 \mathrm{~ns}$ \\
\hline Plongée sous-marine & 40,5 & 32,4 & $8,1^{\star \star}$ & 47,0 & 44,8 & $2,2 \mathrm{~ns}$ \\
\hline Tennis & 32,8 & 23,3 & $9,5^{\star \star}$ & 42,8 & 37,8 & $5,0 \mathrm{~ns}$ \\
\hline Tennis de table & 23,7 & 13,3 & $10,4^{\star \star}$ & 30,7 & 19,8 & $10,9^{*}$ \\
\hline Handball & 25,4 & 14,3 & 11,1 & 34,0 & 22,1 & $11,9^{\star \star}$ \\
\hline Base-ball & 25,1 & 13,6 & $11,5^{\star \star}$ & 30,2 & 19,2 & $11,0^{*}$ \\
\hline
\end{tabular}




\begin{tabular}{|c|c|c|c|c|c|c|}
\hline \multirow{2}{*}{$\begin{array}{l}\text { Activités physiques } \\
\text { Cyclisme }\end{array}$} & \multicolumn{2}{|c|}{$\begin{array}{c}\text { Pratique } \\
\text { souhaitée (tout } \\
\text { l'échantillon) }\end{array}$} & \multirow{2}{*}{$\begin{array}{c}\text { Différences } \\
\text { entre garçons } \\
\text { et filles } \\
11,6^{\star \star}\end{array}$} & \multicolumn{2}{|c|}{$\begin{array}{l}\text { Pratique souhai- } \\
\text { tée (inscrits au } \\
\text { sport scolaire) }\end{array}$} & \multirow{2}{*}{$\begin{array}{c}\text { Différences } \\
\text { entre garçons } \\
\text { et filles } \\
11,5^{\star \star}\end{array}$} \\
\hline & 21,9 & 10,3 & & 28,4 & 16,9 & \\
\hline Hockey & 26,5 & 14,2 & $12,3^{\star \star}$ & 32,6 & 17,4 & $15,2^{* \star}$ \\
\hline Ski alpin & 33,3 & 20,7 & $12,6^{\star \star}$ & 38,1 & 29,1 & $9,0 \mathrm{~ns}$ \\
\hline Musculati & 34,5 & 18,2 & $16,3^{\star \star}$ & 37,7 & 20,9 & $16,8^{\star \star}$ \\
\hline Rugby & 25,2 & 8,2 & $17,0^{\star \star}$ & 33,0 & 16,3 & $16,7^{\star *}$ \\
\hline VTT & 35,9 & 17,6 & $18,3^{\star \star}$ & 40,9 & 25,6 & $15,3^{\text {** }}$ \\
\hline Football & 28,2 & 8,4 & $19,8^{\star \star}$ & 34,9 & 15,1 & $19,8^{* *}$ \\
\hline
\end{tabular}

* Différences significatives à un seuil < ou = à $5 \%$

** Différences significatives à un seuil < ou = à $1 \%$ ns: différences non significatives statistiquement GRS : gymnastique rythmique et sportive 